\title{
Separating the apples from the oranges: from NAFLD heterogeneity to personalized medicine
}

\author{
Amedeo Lonardo*(1) \\ Department of Internal Medicine, Azienda Ospedaliero-Universitaria of Modena, 41100 Modena, Italy
}

*Correspondence: Amedeo Lonardo, Ospedale Civile di Baggiovara,Via Giardini 1135, 41126 Modena, Italy. a.lonardo@libero. it; lonardo.amedeo@aou.mo.it

Academic Editor: Lindsay A. Farrer, Boston University School of Medicine, USA

Received: September 27, 2021 Accepted: October 9, 2021 Published: October 31, 2021

Cite this article: Lonardo A. Separating the apples from the oranges: from NAFLD heterogeneity to personalized medicine. Explor Med. 2021;2:435-42. https://doi.org/10.37349/emed.2021.00061

\begin{abstract}
Recently, Arrese and Colleagues have published a review article entitled, "Insights into Nonalcoholic FattyLiver Disease (NAFLD) Heterogeneity" (Semin Liver Dis. 2021;41:421-34. doi: 10.1055/s-0041-1730927). This milestone publication clearly and exhaustively explains the multitude of pathogenic pathways involved in the development and progression of disease eventually conducive to heterogeneous clinical phenotypes and different disease outcomes. The present commentary first briefly discusses the biological grounds of NAFLD heterogeneity and then illustrates the work by Arrese et al. In conclusion, the presently adopted nomenclatures appear inadequate in rendering the complexity of disease in the individual patient. In order to adopt the principles of personalized care, what remains to be done is to propose and validate a simple and accurate classification system. This should give full consideration to the principal disease modifiers and should shape a scheme to be adopted in both clinical practice and in the research arena. Care should be taken to not neglect the systemic nature of disease.
\end{abstract}

\section{Keywords}

Clinical phenotypes, diagnosis, disease outcomes, heterogeneity, management, natural course, personalized medicine, precision medicine, variability

"NASH is currently a generic diagnosis that encompasses multiple diseases. Precise etiologic definitions are necessary to keep the apples separate from the oranges within the fruit families". Albert J Czaja, 1997

\section{NAFLD definitions, burden and scope}

\section{Nomenclature}

In 1986, Shaffner and Thaler coined the definition of nonalcoholic fatty liver disease (NAFLD) although what we now call NAFLD had previously been reported under different names [1]. Essentially NAFLD describes a clinico-pathological syndrome of variable types of fatty liver changes [from steatosis to nonalcoholic steatohepatitis (NASH) with/without fibrosis, cirrhosis and hepatocellular carcinoma (HCC)] that may occur either primarily or in a complex relationship with various other conditions (Table 1) [2,3].

(C) The Author(s) 2021. This is an Open Access article licensed under a Creative Commons Attribution 4.0 International License (https://creativecommons.org/licenses/by/4.0/), which permits unrestricted use, sharing, adaptation, distribution and reproduction in any medium or format, for any purpose, even commercially, as long as you give appropriate credit to the original author(s) and the source, provide a link to the Creative Commons license, and indicate if changes were made. 
Table 1. Classification of NAFLD [2, 3]

\begin{abstract}
Primary NAFLD
Associated with/predisposing to incident metabolic syndrome

Secondary NAFLD

Nutritional disorders

total parenteral nutrition, acute starvation

Abdominal surgery

extensive small bowel resection bilio-pancreatic diversion, jejunal by-pass

Drug-induced

Occupational exposure to organic solvents

(Rare) Metabolic disorders

hypobetalipoproteinemia, lipodystrophy, Weber-Christian syndrome, acute fatty liver of pregnancy, Reyes syndrome and Mauriac syndrome

VAFLD

HIV, HCV

Common endocrine disorders

hypothyroidism, hypogonadism in either sex, GH deficiency, PCOS

VAFLD: virus-associated fatty liver disease; GH: growth hormone; PCOS: policystic ovary syndrome; HIV: human immunodeficiency virus; $\mathrm{HCV}$ : heptitis $\mathrm{C}$ virus

Note. Reprinted from "Renaming NAFLD to MAFLD: could the LDE system assist in this transition?" by Lonardo A. J Clin Med. 2021;10:492 (https://doi.org/10.3390/jcm10030492). CC BY.

In 2020, a panel of authors proposed that NAFLD should be renamed metabolic-associated fatty liver disease (MAFLD) [4]. However, NAFLD and MAFLD are not entirely equivalent and the modification is more than a single-letter change in an acronym [5]. Additionally, the first and somewhat neglected hint to disease variability is the finding that a reduction of steatosis to the point of complete fat loss may accompany the development of advanced liver fibrosis in NASH [6]. This apparent paradox suggests that even the name "fatty liver" is not invariably appropriate in all NAFLD/MAFLD forms and stages. As such, it seems that we are failing to personalize diagnosis from the very beginning, namely from disease definition.
\end{abstract}

\title{
NAFLD is a clinical and public health load
}

The importance of NAFLD may be envisaged under both a clinical and public health perspective. Clinically, NAFLD poses the challenge of a common condition whose diagnosis, by definition, is based on histological grounds, though no licensed treatment is available [7]. As a public health problem, large strata of global population have one in four individuals suffering from NAFLD, which places a formidable burden on health systems [8].

Aim

Within this complex and worrisome scenario, a milestone article recently published by Arrese and colleagues [9], addresses heterogeneity as a fundamental aspect of NAFLD which dictates, through an extraordinary variability of pathogenic pathways, the development of heterogeneous clinical phenotypes and disease outcomes [9]. The present commentary briefly discusses the bases of NAFLD heterogeneity first and next critically illustrates the work by Arrese et al. [9].

\section{Modifiers in NAFLD epidemiology, pathobiology, clinical aspects and natural course}

\section{Epidemiology and pathobiology}

Epidemiological models have clearly indicated that the principal physiologic modifiers of NAFLD are age, sex and ethnicity, along with belonging to "non-alcoholic fatty liver disease families" and carrying risk alleles 
for selected genetic polymorphisms [10]. Among the metabolic modifiers, metabolic syndrome and its components should be considered as the major determinants of NAFLD risk [10].

Why do some, but not other, individuals with simple hepatic steatosis progress to developing NASH? And, importantly, why do only a fraction of those with NASH progress to cirrhosis? Although we have no definite answer to these questions, from a pathobiological perspective, it is fully conceivable that the heterogeneity of disease course reflects variability in exposure and response to metabolic stress, susceptibility to hepatocyte lipotoxicity, differences in repair-response efficacy and "metabolic inflammation" [11,12]. The net balance among these various pathogenic factors will eventually promote the progression of fibrosis and risk of HCC in NASH [13]. Therefore, the identification of simple biomarkers identifying and scoring each of the above pathomechanisms is a major challenge in translational research.

\section{Natural course and clinical aspects}

Studies evaluating biopsy-proven disease suggest that progressive fibrosis develops in up to about 50\% of NASH cases, with advanced fibrosis/cirrhosis eventually ensuing in about $10 \%$ of the overall NAFLD population [14]. Among NAFLD patients, the only histological independent predictor of liver-related morbidity/mortality, and mortality owing to all-causes, is liver fibrosis, not NASH [14,15]. However, individual patients exhibit a striking variability in disease progression owing to alcohol consumption, obesity, type 2 diabetes/insulin resistance and genetic factors all playing a key role as disease modifiers [16].

The multiplicity of pathogenic pathways involved in disease progression dictate a substantial heterogeneity of clinical phenotypes of NAFLD and also affect and hinder the diagnostic process [14, 17]. Schematically, a "hepatic NAFLD" form exists as opposed to an "extra-hepatic NAFLD", the latter accounting for the leading cause of mortality, namely cardiovascular and cancer-related and the former following the course of all chronic liver diseases owing to other etiologies [18]. The confluence, in variable proportions, of the two principal NAFLD forms may ultimately contribute to shaping a potentially uncountable variety of clinical phenotypes and disease outcomes.

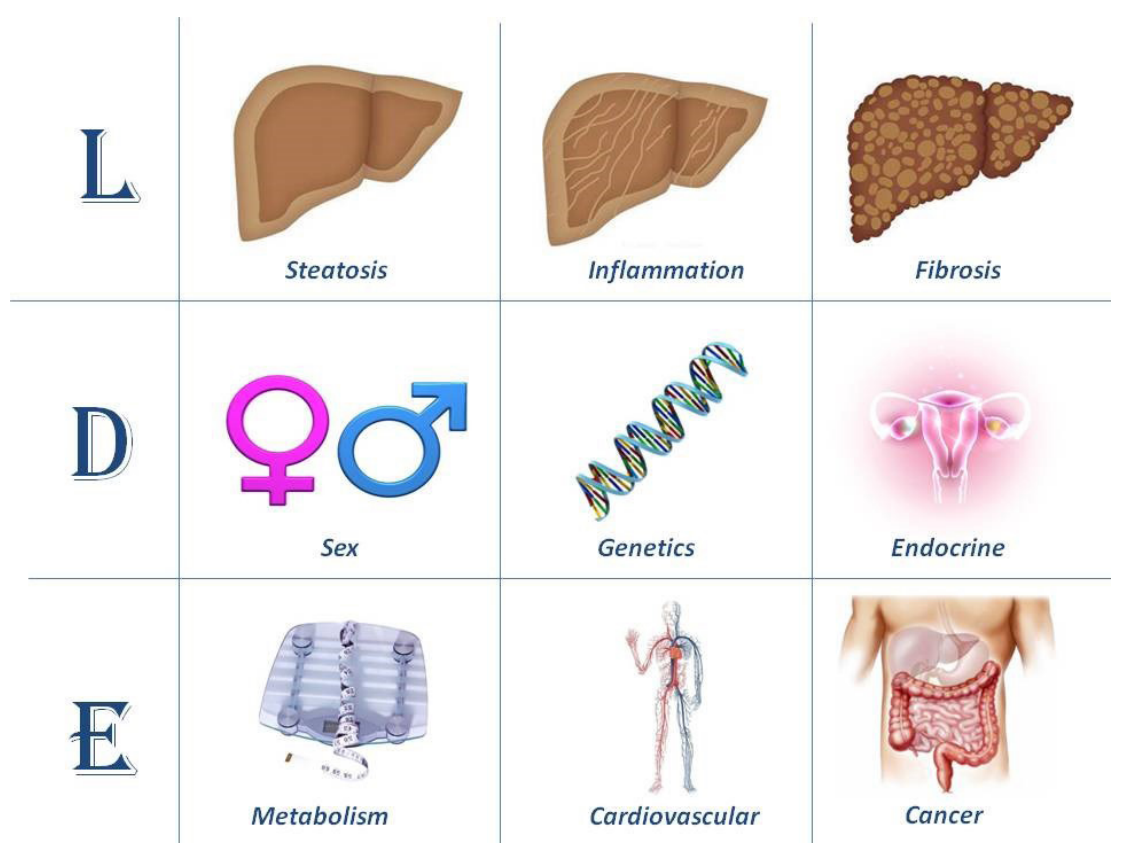

Figure 1. The LDE system [2, 19]. In its essence, the LDE system requires clarification of nine criteria that should be recorded to better characterize cohorts of patients. The prefix "L" for "Liver" aims at analytically illustrating the severity of liver involvement based on either non-invasive or histological assessment. Cirrhosis and/or HCC should be declared here. The central part of the LDE system is " $D$ " for "determinants". Among these, sex and reproductive status must be emphasized and genetic determinants should also be acknowledged, if available. Type 2 diabetes/insulin resistance and obesity should always be confirmed/excluded. Hypothyroidism should also be evaluated. Other endocrine derangements, such as polycystic ovary syndrome, growth hormone deficiency and hypogonadism (in both sexes), should always be declared. The suffix "E" for "extra-hepatic" reports information regarding non-hepatic, i.e. systemic manifestations of disease

Note. Reprinted from "Renaming NAFLD to MAFLD: could the LDE system assist in this transition?" by Lonardo A. J Clin Med. 2021;10:492 (https://doi.org/10.3390/jcm10030492). CC BY. 
Today, little has been proposed to translate the above clinical and pathobiological premises into a consistent and feasible categorization scheme. However, the so called "LDE" classification system (Figure 1) [2,19] or similar systematic approaches, may eventually lead to selecting more homogenous subgroups of patients with comparable natural course and predictable response to therapy in the setting of advancing the frontiers of personalized NAFLD medicine [20].

\section{The article by Arrese and colleagues}

In the setting of those unmet needs to identify innovative and effective treatment strategies, it has previously been suggested that personalization of diagnosis and management of disease can lay the grounds for more individualized therapeutic plans [20]. A recent article by Arrese et al. [9] offers novel insights into NAFLD heterogeneity. The article is elegantly shaped and structured so as to comprehensively cover the role of age, sex, hormonal and reproductive status; genetics; epigenetics and ethnicity; bodily composition (muscle mass and intestinal microbiome); metabolic health and lifestyle habits (e.g., alcohol consumption, physical activity). Next, the diagnostic and therapeutic bearings of the above modifiers are illustrated.

Regarding the proposal to rename NAFLD to MAFLD the authors feel that "the use of MAFLD may be a step forward in addressing disease heterogeneity by grouping a more homogeneous patient population with more severe metabolic and liver disease" [9]. However, voicing published comments and proposals, Arrese and colleagues [9] also correctly point out that MAFLD might/should be better articulated by identifying at least individuals with lean MAFLD, obese MAFLD, diabetic MAFLD as well as cases of MAFLD with significant fibrosis.

\section{Commentary and research agenda}

Taken collectively, the data summarized above, together with the paper by Arrese et al. [9], indicate that epidemiological, pathobiological and clinical perspectives consistently converge in identifying the complex array of physiologic-lifestyle modifiers, endocrine-metabolic variables and pathogenic-histological features that act as disease modifiers in the NAFLD/MAFLD arena. However, both NAFLD and MAFLD appear to be inadequate definitions in rendering the complexity of disease in the individual patient [19-21].

In the attempt to adopt the principles of personalized care [22], what remains to be done is to propose and validate a simple and accurate classification system. This should take into account the principal, if not all, of the previously discussed disease variables to devise a practical categorization scheme to be adopted in clinical practice and in the research arena. In doing so, particular care should be exercised to not neglect the systemic nature of disease. Considering that the leading causes of mortality include cardiovascular events and cancer [18], NAFLD patients should be comprehensively classified based also on their prominent extrahepatic features rather than on their "intra-hepatic" manifestations alone [23-25]. Table 2 [26-42] summarizes some selected topics of future research. In conclusion, in 1997, the distinguished liver immunologist Albert J Czaja wrote that "NASH is currently a generic diagnosis that encompasses multiple diseases. Precise etiologic definitions are necessary to keep the apples separate from the oranges within the fruit families" [43]. His illuminated words are the best synthesis of what, many years later, still remains to be done.

Table 2. Research agenda of selected topics on precision medicine approaches to NAFLD

\begin{tabular}{llc}
\hline Diagnostic and physiopathological characterization & Ref. \\
\hline Non-invasive & Biomarkers of oxidative stress and antioxidant status & {$[26,27]$} \\
& Lipid signature including lysophospholipids, bile acids and biomarkers & {$[28-30]$} \\
of subtypes of deranged lipid metabolism & {$[31]$} \\
& miRNAs & {$[32]$} \\
& Identification of metabolite biomarkers & Intestinal microbiome-based personalized approaches can be used \\
& as a non-invasive biomarker for NAFLD diagnosis and staging, \\
\hline
\end{tabular}


Table 2. Research agenda of selected topics on precision medicine approaches to NAFLD (continued)

\begin{tabular}{|c|c|c|}
\hline \multicolumn{2}{|c|}{ Diagnostic and physiopathological characterization } & \multirow{2}{*}{ Ref. } \\
\hline Based on analysis of liver samples & $\begin{array}{l}\text { Multi-omics integrative study of liver tissue-specific genes, processes, } \\
\text { and networks }\end{array}$ & \\
\hline & $\begin{array}{l}\text { Identification of patient-specific } \\
\text { activation of morphogenic pathways known to affect zonal development } \\
\text { and maintenance, and nuclear hormone receptors downstream of } \\
\text { potential NASH therapeutics }\end{array}$ & [35] \\
\hline \multirow[t]{6}{*}{ Management } & Personalized diet and physical activity/exercise & [36] \\
\hline & $\begin{array}{l}\text { Personalized restoration of the intestinal ecosystem with engineered } \\
\text { bacterial strains that produce anti-inflammatory peptides, or beneficial } \\
\text { antioxidants, or drugs that either inhibit or mimic specific bacterial } \\
\text { enzymes; fecal microbiota transplantation }\end{array}$ & [36-38] \\
\hline & Patient-derived induced pluripotent stem cells & [39] \\
\hline & $\begin{array}{l}\text { Antisense oligonucleotides to target mature miRNAs to reduce their } \\
\text { biologic effect, as well as the use of viral vectors to deliver miRNA } \\
\text { erasers, which would bind miRNA targets competitively but without } \\
\text { producing the biological effect }\end{array}$ & {$[31,40]$} \\
\hline & Pharmacogenomics & [41] \\
\hline & $\begin{array}{l}\text { Targeting of the signaling controls of key genes implicated in the } \\
\text { development of NAFLD }\end{array}$ & {$[40,42]$} \\
\hline
\end{tabular}

\section{Abbreviations}

HCC: hepatocellular carcinoma

MAFLD: metabolic-associated fatty liver disease

NAFLD: nonalcoholic fatty liver disease

NASH: nonalcoholic steatohepatitis

\section{Declarations}

Acknowledgment

I am indebted to my wife, Jacqueline Mole, for editing English carefully.

\section{Author contributions}

The author contributed solely to the work.

\section{Conflicts of interest}

The author declares that he has no conflicts of interest.

\section{Ethical approval}

Not applicable.

\section{Consent to participate}

Not applicable.

\section{Consent to publication}

Not applicable.

\section{Availability of data and materials}

Not applicable.

\section{Funding}

Not applicable. 


\section{Copyright}

(C) The Author(s) 2021.

\section{References}

1. Lonardo A, Leoni S, Alswat KA, Fouad Y. History of nonalcoholic fatty liver disease. Int J Mol Sci. 2020;21:5888.

2. Lonardo A. Renaming NAFLD to MAFLD: could the LDE system assist in this transition? J Clin Med. 2021;10:492.

3. Liebe R, Esposito I, Bock HH, Vom Dahl S, Stindt J, Baumann U, et al. Diagnosis and management of secondary causes of steatohepatitis. J Hepatol. 2021;74:1455-71.

4. Eslam M, Newsome PN, Sarin SK, Anstee QM, Targher G, Romero-Gomez M, et al. A new definition for metabolic dysfunction-associated fatty liver disease: an international expert consensus statement. J Hepatol. 2020;73:202-9.

5. Targher G. From nonalcoholic fatty liver disease to metabolic dysfunction-associated fatty liver disease: more than a single-letter change in an acronym. Hepatoma Res. 2021;7:47.

6. van der Poorten D, Samer CF, Ramezani-Moghadam M, Coulter S, Kacevska M, Schrijnders D, et al. Hepatic fat loss in advanced nonalcoholic steatohepatitis: are alterations in serum adiponectin the cause? Hepatology. 2013;57:2180-8.

7. European Association for the Study of the Liver (EASL); European Association for the Study of Diabetes (EASD); European Association for the Study of Obesity (EASO). EASL-EASD-EASO Clinical Practice Guidelines for the management of non-alcoholic fatty liver disease. Diabetologia. 2016;59:1121-40.

8. Younossi ZM. Non-alcoholic fatty liver disease - a global public health perspective. J Hepatol. 2019;70:531-44.

9. Arrese M, Arab JP, Barrera F, Kaufmann B, Valenti L, Feldstein AE. Insights into nonalcoholic fatty-liver disease heterogeneity. Semin Liver Dis. 2021;41:421-34.

10. Non-alcoholic Fatty Liver Disease Study Group; Lonardo A, Bellentani S, Argo CK, Ballestri S, Byrne CD, Caldwell SH, et al. Epidemiological modifiers of non-alcoholic fatty liver disease: focus on high-risk groups. Dig Liver Dis. 2015;47:997-1006.

11. Suzuki A, Diehl AM. Nonalcoholic steatohepatitis. Annu Rev Med. 2017;68:85-98.

12. Katsarou A, Moustakas II, Pyrina I, Lembessis P, Koutsilieris M, Chatzigeorgiou A. Metabolic inflammation as an instigator of fibrosis during non-alcoholic fatty liver disease. World J Gastroenterol. 2020;26:1993-2011.

13. Loomba R, Friedman SL, Shulman GI. Mechanisms and disease consequences of nonalcoholic fatty liver disease. Cell. 2021;184:2537-64.

14. Pais R, Maurel T. Natural history of NAFLD. J Clin Med. 2021;10:1161.

15. Calzadilla Bertot L, Adams LA. The natural course of non-alcoholic fatty liver disease. Int J Mol Sci. 2016;17:774.

16. Nasr P, Blomdahl J, Kechagias S, Ekstedt M. Modifiers of liver-related manifestation in the course of NAFLD. Curr Pharm Des. 2020;26:1062-78.

17. Wentworth BJ, Caldwell SH. Pearls and pitfalls in nonalcoholic fatty liver disease: tricky results are common. Metab Target Organ Damage. 2021;1:2.

18. Italian Association for the Study of the Liver (AISF). AISF position paper on nonalcoholic fatty liver disease (NAFLD): updates and future directions. Dig Liver Dis. 2017;49:471-83.

19. Lonardo A, Ballestri S. Perspectives of nonalcoholic fatty liver disease research: a personal point of view. Explor Med. 2020;1:85-107. 
20. Lonardo A, Arab JP, Arrese M. Perspectives on precision medicine approaches to NAFLD diagnosis and management. Adv Ther. 2021;38:2130-58.

21. Lonardo A. Back to the future. From the history of NAFLD to MAFLD to heterogeneity of disease. Clin Transl Discovery. Forthcoming 2021.

22. Lonardo A, Byrne CD and Targher G. Precision medicine approaches in metabolic disorders and target organ damage: where are we now, and where are we going? Metab Target Organ Damage. 2021;1:3.

23. Mantovani A, Scorletti E, Mosca A, Alisi A, Byrne CD, Targher G. Complications, morbidity and mortality of nonalcoholic fatty liver disease. Metabolism. 2020;111S:154170.

24. Sanna C, Rosso C, Marietti M, Bugianesi E. Non-alcoholic fatty liver disease and extra-hepatic cancers. Int J Mol Sci. 2016;17:717.

25. Pellicori P, Vaduganathan M, Ferreira JP, Zannad F, Sanyal AJ. Cross-talk between non-alcoholic fatty liver disease and cardiovascular disease: implications for future trial design. Diabetes Metab. 2021;[Epub ahead of print].

26. Martinez-Urbistondo D, Suarez Del Villar R, Argemí J, Daimiel L, Ramos-López O, San-Cristobal R, et al. Antioxidant lifestyle, co-morbidities and quality of life empowerment concerning liver fibrosis. Antioxidants (Basel). 2020;9:1125.

27. Delli Bovi AP, Marciano F, Mandato C, Siano MA, Savoia M, Vajro P. Oxidative stress in non-alcoholic fatty liver disease. An updated mini review. Front Med (Lausanne). 2021;8:595371.

28. Christinat N, Valsesia A, Masoodi M. Untargeted profiling of bile acids and lysophospholipids identifies the lipid signature associated with glycemic outcome in an obese non-diabetic clinical cohort. Biomolecules. 2020;10:1049.

29. Mato JM, Alonso C, Noureddin M, Lu SC. Biomarkers and subtypes of deranged lipid metabolism in nonalcoholic fatty liver disease. World J Gastroenterol. 2019;25:3009-20.

30. Jiao N, Loomba R, Yang ZH, Wu D, Fang S, Bettencourt R, et al. Alterations in bile acid metabolizing gut microbiota and specific bile acid genes as a precision medicine to subclassify NAFLD. Physiol Genomics. 2021;53:336-48.

31. Mahgoub A, Steer CJ. MicroRNAs in the evaluation and potential treatment of liver diseases. J Clin Med. 2016;5:52.

32. Nielsen J. Systems biology of metabolism: a driver for developing personalized and precision medicine. Cell Metab. 2017;25:572-9.

33. Sharpton SR, Schnabl B, Knight R, Loomba R. Current concepts, opportunities, and challenges of gut microbiome-based personalized medicine in nonalcoholic fatty liver disease. Cell Metab. 2021;33:21-32.

34. Kurt Z, Barrere-Cain R, LaGuardia J, Mehrabian M, Pan C, Hui ST, et al. Tissue-specific pathways and networks underlying sexual dimorphism in non-alcoholic fatty liver disease. Biol Sex Differ. 2018;9:46.

35. Steinman JB, Salomao MA, Pajvani UB. Zonation in NASH - a key paradigm for understanding pathophysiology and clinical outcomes. Liver Int. 2021;41:2534-46.

36. Houghton D, Stewart CJ, Day CP, Trenell M. Gut microbiota and lifestyle interventions in NAFLD. Int J Mol Sci. 2016;17:447.

37. Bluemel S, Williams B, Knight R, Schnabl B. Precision medicine in alcoholic and nonalcoholic fatty liver disease via modulating the gut microbiota. Am J Physiol Gastrointest Liver Physiol. 2016;311:G1018-36.

38. Hartmann P, Schnabl B. New developments in microbiome in alcohol-associated and nonalcoholic fatty liver disease. Semin Liver Dis. 2021;41:87-102.

39. Chien Y, Huang CS, Lin HC, Lu KH, Tsai PH, Lai YH, et al. Improvement of non-alcoholic steatohepatitis by hepatocyte-like cells generated from iPSCs with Oct4/Sox2/Klf4/Parp1. Oncotarget. 2018;9:18594-606. 
40. Murray JK, Long J, Liu L, Singh S, Pruitt D, Ollmann M, et al. Identification and optimization of a minor allele-specific small interfering RNA to prevent PNPLA3 I148M-driven nonalcoholic fatty liver disease. Nucleic Acid Ther. 2021;31:324-40.

41. Lorbek G, Urlep Ž, Rozman D. Pharmacogenomic and personalized approaches to tackle nonalcoholic fatty liver disease. Pharmacogenomics. 2016;17:1273-88.

42. Schwartz BE, Rajagopal V, Smith C, Cohick E, Whissell G, Gamboa M, et al. Discovery and targeting of the signaling controls of PNPLA3 to effectively reduce transcription, expression, and function in pre-clinical NAFLD/NASH settings. Cells. 2020;9:2247.

43. Czaja AJ. Recurrence of nonalcoholic steatohepatitis after liver transplantation. Liver Transpl Surg. 1997;3:185-6. 